\title{
Exciton Dynamics in Colloidal Quantum-Dot LEDs under Active Device Operations
}

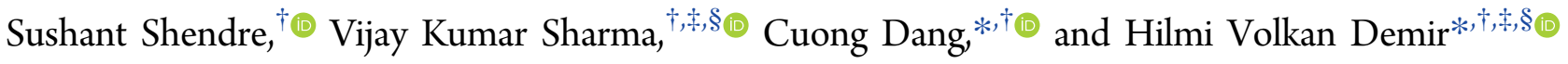 \\ ${ }^{\dagger}$ LUMINOUS! Centre of Excellence for Semiconductor Lighting and Displays, School of Electrical and Electronic Engineering, The \\ Photonics Institute (TPI), Nanyang Technological University, Singapore 639798 \\ ${ }^{\ddagger}$ School of Physical and Mathematical Sciences, Nanyang Technological University, Singapore 639798 \\ ${ }^{\S}$ Department of Electrical and Electronics Engineering and Department of Physics, UNAM-Institute of Materials Science and \\ Nanotechnology, Bilkent University, Bilkent, Ankara, Turkey 06800
}

\section{Supporting Information}

ABSTRACT: Colloidal quantum-dot light-emitting diodes (QLEDs) are lucrative options for color-pure lighting sources. To achieve high-performance QLEDs, besides developing highefficiency quantum dots (QDs), it is essential to understand their device physics. However, little understanding of the QD emission behavior in active QLEDs is one of the main factors hindering the improvement of device efficiency. In this work, we systematically studied the exciton dynamics of gradient composition CdSe@ZnS QDs during electroluminescence in a working QLED. With timeresolved photoluminescence analyses using fluorescence lifetime imaging microscopy we analyzed a large population of QDs spatially spreading over an extended area inside and outside the device. This allows us to reveal the statistically significant changes in the behavior of $\mathrm{QD}$ emission in the device at different levels of applied voltages and injection currents. We find that the QD emission efficiency first drops in device fabrication with $\mathrm{Al}$ electrode deposition and that the $\mathrm{QD}$ exciton lifetime is then statistically reduced further under the QLED's working conditions. This implies the nonradiative Auger recombination process is active in charged QDs as a result of imbalanced charge injection in a working QLED. Our results help to understand the exciton behavior during the operation of a QLED and demonstrate a new approach to explore the exciton dynamics statistically with a large QD population.

KEYWORDS: exciton dynamics, electroluminescence, semiconductor nanocrystals, colloidal quantum dots, light-emitting diodes, Auger recombination
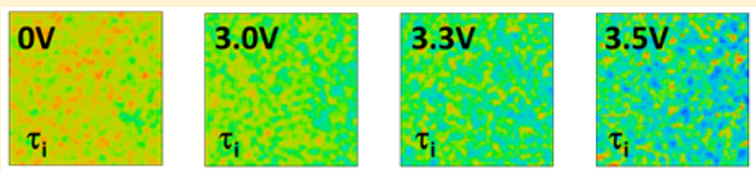

홀

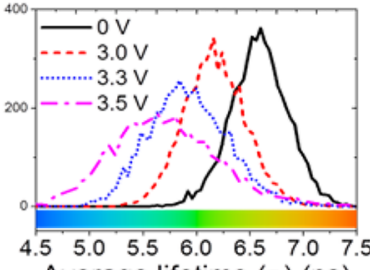

Average lifetime $\left(\tau_{\mathrm{i}}\right)(\mathrm{ns})$ olloidal semiconductor nanocrystals or quantum dots

(QDs) are well known for their potentials of being excellent materials for lighting and display technologies. ${ }^{1-6}$ They combine the advantages of low-cost colloidal chemistry synthesis, the stability of inorganic semiconductor compounds, and the wide band-gap tunability of nanomaterials. For optoelectronic applications, QDs have been used as downconverters for current solid-state lighting and displays, emissive layers in light-emitting diodes (QLEDs), ${ }^{7-10}$ and optical gain media in lasers of multiple colors. ${ }^{11-13}$ Studies on single QDs and their ensemble behavior have given insight into the factors affecting the electro- and photoluminescence of these materials. ${ }^{14-17}$ Recent advances in colloidal synthesis techniques have enabled production of QDs with near-unity photoluminescence (PL) quantum yields (QYs), ${ }^{18}$ with high robustness in different environments ${ }^{19}$ or with engineered structures to fine-tune their quantum electronic properties such as suppressed Auger recombination rates. ${ }^{20,21}$ For example, a thick shell and an alloyed core-shell interface for QDs were shown to be successful in the suppression of Auger recombination and resulted in high quantum yields. ${ }^{21-23}$

With the rapid progress in the development of high-quality QDs, it became possible to develop high-performance QLEDs with near $100 \%$ internal quantum efficiency (IQE)., However, in many cases, the QDs usually do not maintain their performance under device operating conditions, and thus the QLEDs suffer from an efficiency roll-off behavior at high current densities. The limiting factors of efficient operation of QLEDs are being actively studied to understand and improve their performance not only on the efficiency but also on the intensity and the stability. ${ }^{24-26}$ Initially, Shirasaki et $\mathrm{al}^{27}$ reported that efficiency roll-off in QLEDs is electric field induced and is not related to QD charging. Bozygit et al. ${ }^{28}$ also suggested the electric field induced quantum-confined Stark effect (QCSE) to be the primary cause. However, in the same

Received: August 30, 2017

Published: November 8, 2017 
a)

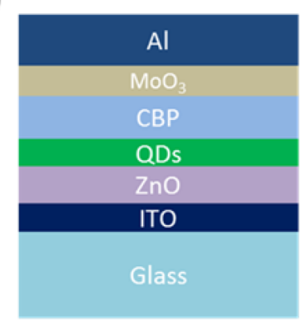

b)

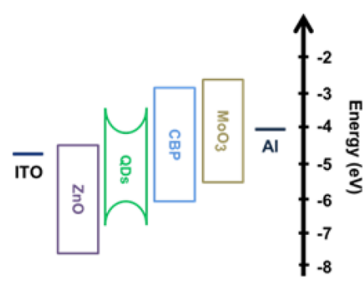

d)

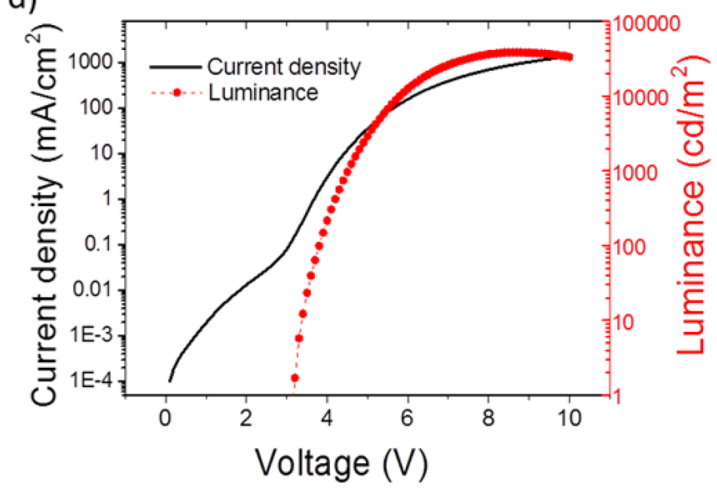

c)

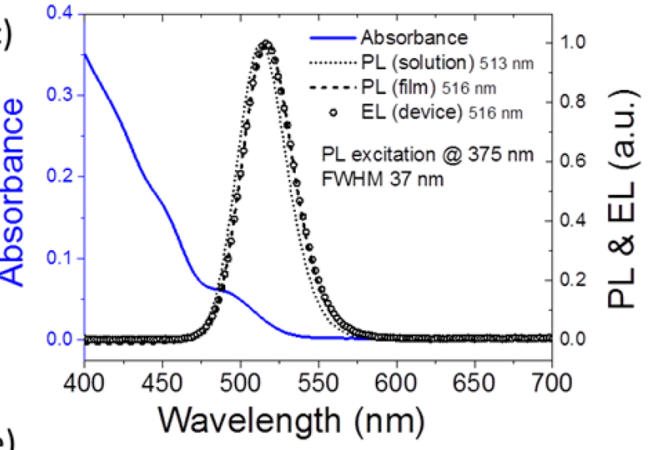

e)

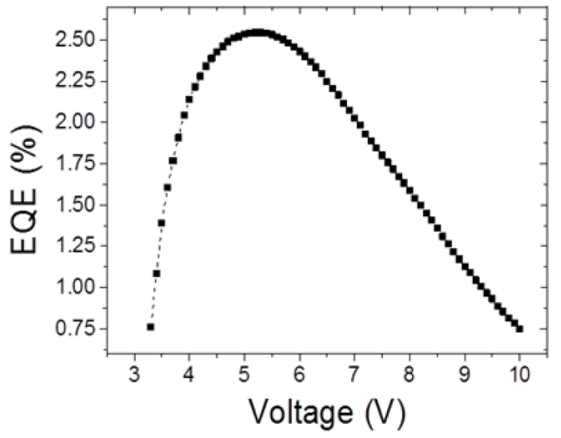

Figure 1. (a) Schematic diagram of the QLED structure. (b) Zero bias energy band configuration of the device layers. (c) Absorbance, photoluminescence, and electroluminescence spectra. (d) Current density-voltage-luminance $(J-V-L)$ characteristics for the QLED. (e) External quantum efficiency (EQE) variation of the QLED as a function of the bias voltage.

year, Bae et al. $^{29}$ demonstrated that QD charging leads to efficiency roll-off at high current densities by doing timeresolved photoluminescence (TRPL) studies with $\mathrm{CdSe} / \mathrm{CdS}$ C/S QDs. The efficiency roll-off or "droop" has also been reported $^{30-32}$ in GaN-based LEDs explained by charging and the Auger process. They also demonstrated that by introducing an optimized alloy layer between the core and the shell rather than abruptly terminating boundaries, the Auger recombination can be suppressed. It can however be understood that the effect of the electric field (QCSE) is higher in CdSe/CdS QDs when compared with gradient composition CdSe@ZnS QDs. Indeed, development of gradient composition QDs for green-, blue-, and red-emitting QDs of CdSe@ZnS with varying precursor composition showed high-performance QLEDs. ${ }^{33}$ However, there is still not a clear understanding of CdSe@ZnS QD performance in the active QLED and their role in device efficiency. In this work, we systematically studied exciton dynamics of CdSe@ZnS QDs in QLEDs under operating conditions, i.e., during actual electroluminescence. We analyzed the exciton behavior for a significantly large number of QLED regions to understand their dynamics statistically and then correlate them with QLED fabrication and different operating conditions.

\section{RESULTS AND DISCUSSION}

In this work, we fabricated QLED devices with the inverted structure architecture (Figure 1(a) and (b)) using colloidally synthesized green CdSe@ZnS QDs having a gradient composition $\mathrm{Cd}_{1-x} \mathrm{Zn}_{x} \mathrm{Se}_{1-y} \mathrm{~S}_{y}$ structure where $\mathrm{CdSe}$ was at the central core and $\mathrm{ZnS}$ at the outermost shell. ${ }^{34}$ The TEM image of these QDs shows a uniform size $(\sim 6 \mathrm{~nm})$ dispersity (see Figure S1 in the Supporting Information).The absorbance and PL spectra of the QDs are shown in Figure 1(c). The PL of the QD solution has a peak at $513 \mathrm{~nm}$, a full-width at half- maximum (fwhm) of $36 \mathrm{~nm}$, and a QY of $60 \%$ measured with an integrating sphere as the ratio of absolute emission photons and absorption photons. The spin-casted QD films on the glass substrate show a slightly red-shifted spectrum with a $516 \mathrm{~nm}$ peak and a PLQY of $30 \%$. The red shift of the PL spectrum and lower PLQY of the QD film compared to that of the QD solution are known to be associated with the close-packed QDs in the film form. ${ }^{17}$

The device fabrication was started with spin-casting a $\mathrm{ZnO}$ nanoparticle layer of $50 \mathrm{~nm}$ as an electron injection layer on top of a patterned indium tin oxide (ITO) glass. Then, a QD layer of $40 \mathrm{~nm}$ was deposited also by the spin-casting technique. Each spin-casting step was followed by baking at $90{ }^{\circ} \mathrm{C}$ in a nitrogen environment. The sample was then loaded into a thermal evaporator to deposit CBP (4,4'-bis(9-carbazolyl)-1,1'biphenyl, $60 \mathrm{~nm})$ and $\mathrm{MoO}_{3}(10 \mathrm{~nm})$ as the hole transport and injection layer, respectively. The device was finally finished with a $200 \mathrm{~nm}$ thick aluminum (Al) layer as the anode. A schematic structure and the zero bias energy band configurations of the device layers are presented in Figure 1(a) and (b).

The electroluminescence spectrum of our QLED illustrated in Figure 1(c) shows a pure emission of the QD layer at a peak wavelength of $516 \mathrm{~nm}$ and with a $37 \mathrm{~nm}$ fwhm, which are very much similar to the PL characteristics of QDs in the film shown in the same figure. The current density and luminance of the device as functions of the applied bias are presented in Figure 1(d). The luminance reaches the maximum value of $38500 \mathrm{~cd} /$ $\mathrm{m}^{2}$ at a bias of $8.7 \mathrm{~V}$. The turn-on voltage of $3.1 \mathrm{~V}$ is relatively low, implying that the electron and hole were efficiently injected into the emissive QD layer.

The QLED external quantum efficiency (EQE) variation with bias is shown in Figure 1(e). The device reaches a maximum EQE of $2.5 \%$ at around $5.5 \mathrm{~V}$ and rolls-off on increasing the bias. We aim to study the exciton dynamics of 

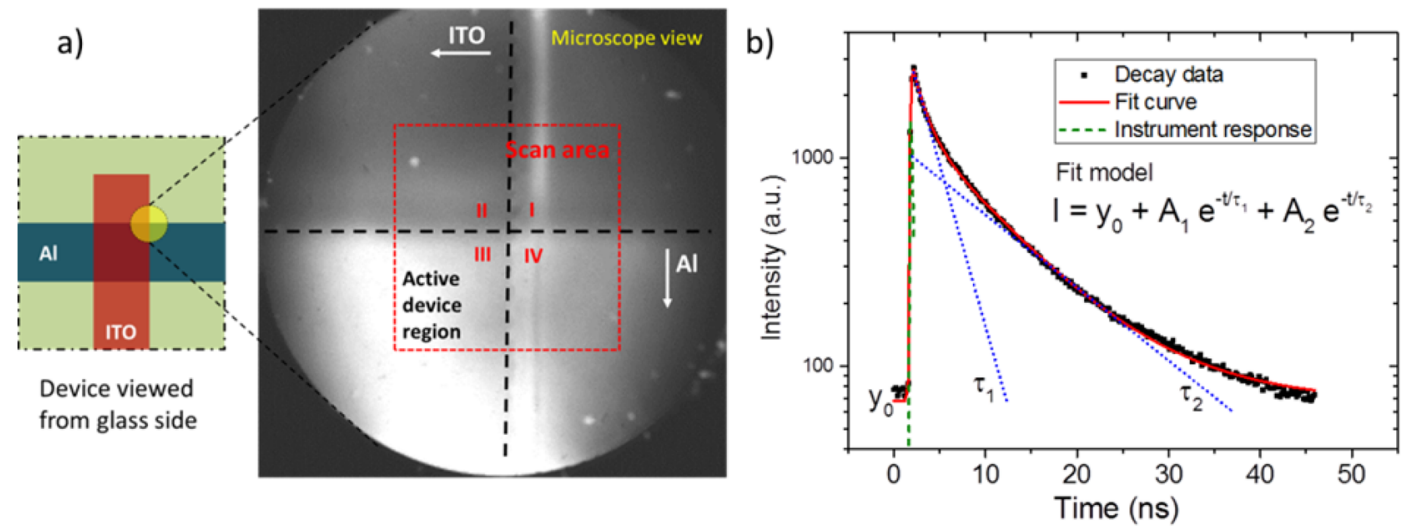

c)

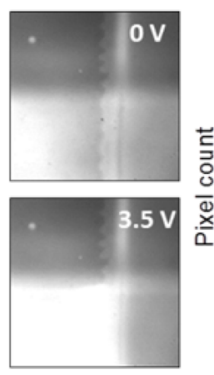

d)

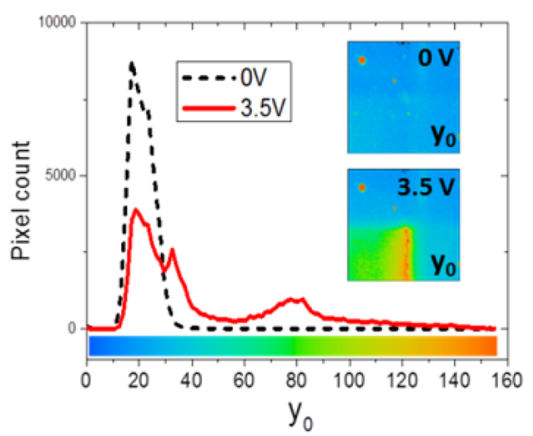

e)

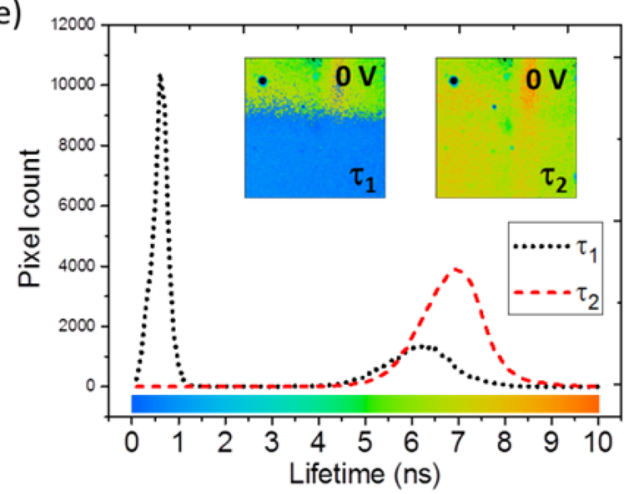

Figure 2. (a) Microscope view of scanned area at the corner of the device. (b) Typical time-resolved photoluminescence decay curve with a twoexponential $\left(\tau_{1}, \tau_{2}\right)$ parameter fit. (c) Intensity images of the scanned area $(256 \times 256$ pixels $)$ at 0 and $3.5 \mathrm{~V}$. (d) Distribution and color-coded images of offset $\left(y_{0}\right)$ in the scan area at 0 and $3.5 \mathrm{~V}$. (e) Distribution and color-coded images of lifetimes $\left(\tau_{1}, \tau_{2}\right)$ in the scan area at $0 \mathrm{~V}$.

the QDs under the device operating conditions, which might provide insights into understanding the reasons for the decrease in efficiency in our QLEDs at high biases.

We use the technique of fluorescence lifetime imaging measurements (FLIM) ${ }^{35}$ to take the time-resolved photoluminescence decay of QDs inside a device under different biasing conditions. Figure 2(a) shows the intensity image captured by time-correlated single photon counting (TCSPC) at the corner of a device. The area of measurement was chosen such that it contains four regions (as illustrated in the figure, quadrants I-IV). The quadrant I consists of the charge transport and emissive layer stack in the device without any of the electrodes on the top and in the bottom. The quadrant II is the region where there is an ITO electrode below the device stack layers. The quadrant III is the active device region formed by the overlap of the $\mathrm{Al}$ and ITO electrodes on the top and in the bottom of the other device layers, respectively. Lastly, the quadrant IV has only the Al electrode on top of the other device layers. The area marked by the red border was used as a scan area on the device, which is about $160 \times 160 \mu \mathrm{m}^{2}$ in area divided into a $256 \times 256$ pixel array. The FLIM measurement consists of recording the time-resolved photoluminescence decay curves in each pixel using the TCSPC technique. Each pixel acts as an ensemble of QDs; thus a FLIM measurement helps us analyze a large number of ensembles of QDs at one time to obtain a statistical distribution of the results.

A typical TRPL decay curve is shown in Figure 2(b). Fitting is applied to each pixel with a two-exponential decay function as shown in the figure $\left(I=y_{0}+A_{1} \mathrm{e}^{-t / \tau}{ }_{1}+A_{2} \mathrm{e}^{-t / \tau}\right)$. Here $y_{0}$ represents the background illumination coming from stray light sources or in our case the electroluminescence from the device after turn-on; $\tau_{1}$ and $\tau_{2}$ are the decay lifetimes, and $A_{1}$ and $A_{2}$ are their corresponding amplitude factors. Figure $2(\mathrm{c})$ shows the intensity images of the scan area at 0 and $3.5 \mathrm{~V}$. On careful observation in the intensity image at $0 \mathrm{~V}$, one can clearly mark out the ITO edge running vertically down. If we divide the image into four quadrants as shown in Figure 2(a), there is a clear contrast in the PL intensities of the pixels in the upper half and lower half of the image. The quadrants III and IV (lower half) have an Al layer, and their higher PL intensity might possibly be due to an optical reflection effect from $\mathrm{Al}$ for both excitation and emission photons. The quadrant III forms the active device region whose electroluminescence can be clearly observed from the intensity image of the scan area at $3.5 \mathrm{~V}$ bias, higher than the device's turn-on voltage of $3.1 \mathrm{~V}$. The single bright spot in the quadrant II appearing in all images is a contaminated spot in our optical measurement system; it is not from the QLED sample and hence is to be removed in our analysis.

By fitting the PL decay curves in each pixel, the decay parameters associated with optoelectronic characteristics of the materials and devices can be extracted. The distributions of these characteristics over the scan area are presented as corresponding color-coded images. Figure 2(d) shows the color-coded images of the parameter $y_{0}$ for the scan area (inset) and its distribution across the pixels at 0 and $3.5 \mathrm{~V}$. Comparing with the intensity images shown in Figure 2(c), we can see that at $0 \mathrm{~V}$ the background intensity in the experiment (i.e., the dark count of the detector, the background photons of the lab) is almost uniform across the scan area with a narrow distribution (in the blue color region). In contrast at $3.5 \mathrm{~V}$, the $y_{0}$ parameter in the active device region (quadrant III) is higher compared to 
a)
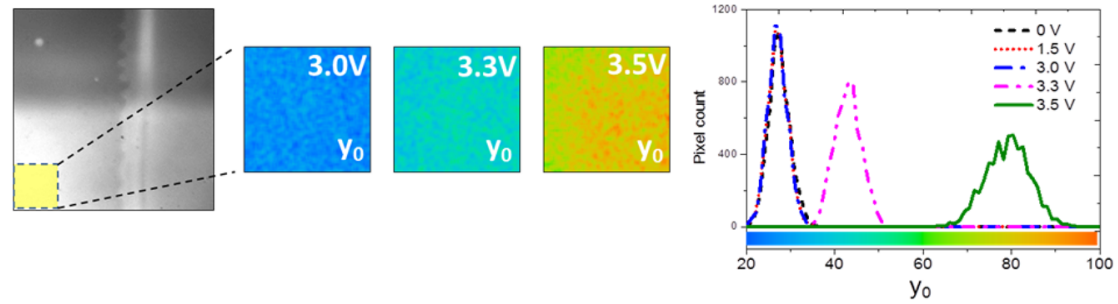

b)

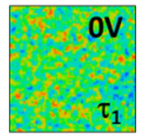

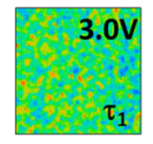
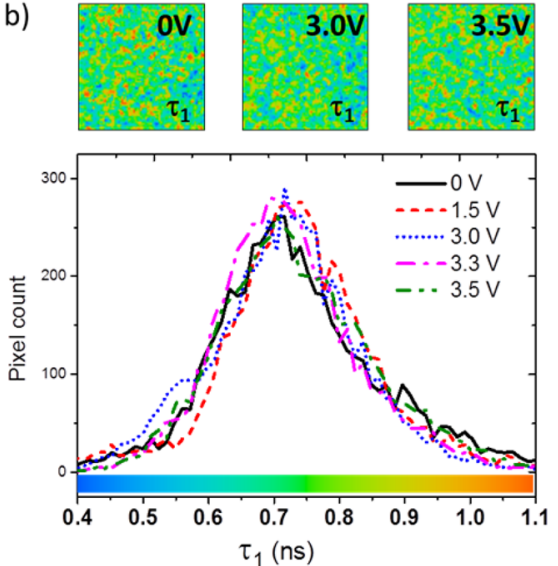

d)

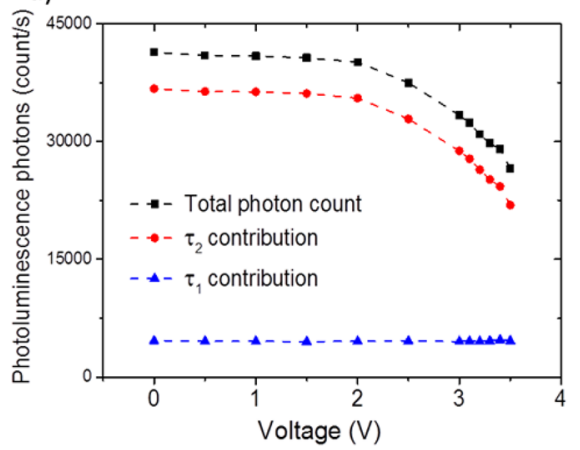

c)
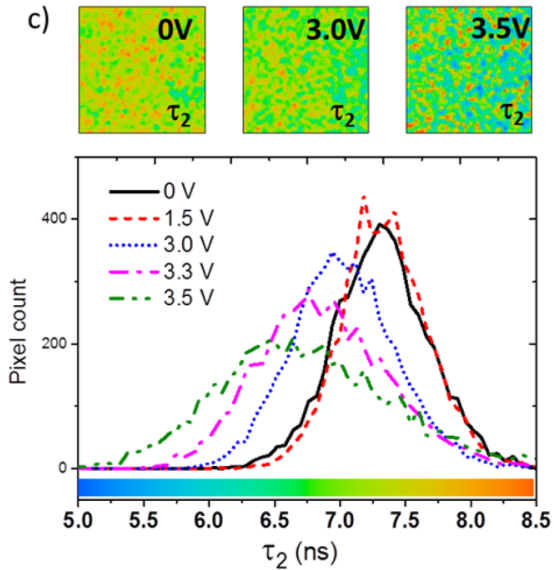

e)

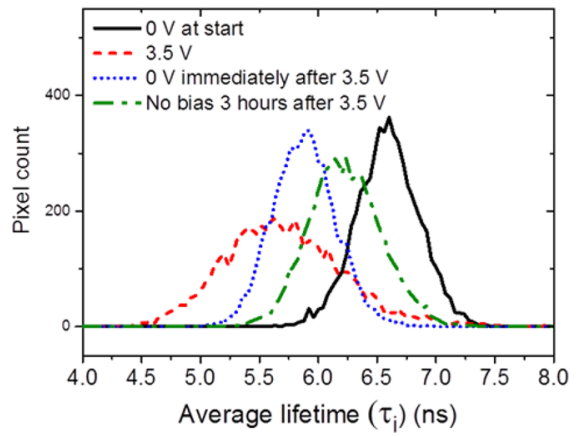

Figure 3. (a) Color-coded images and distribution of offset $\left(y_{0}\right)$ in a small region of the active device in the scan area at different applied biases. (b) Distribution and color-coded images of lifetime $\tau_{1}$ of the same region as shown in (a) at different biases. (c) Distribution and color-coded images of a lifetime $\left(\tau_{2}\right)$ of the same region as shown in (a) at different bias. (d) Photoluminescence photon count of the two lifetime components at different biases in the same region as shown in (a). (f) Intensity-weighted average lifetime $\left(\tau_{\mathrm{i}}\right)$ distribution in the same region as shown in (a) at different times in the experiment.

the other regions due to the electroluminescence from the device adding to the background intensity. This can also be confirmed from the broadened distribution with multiple peaks (in the blue and green color regions) in Figure 2(d). In addition, it shows that the electroluminescence is much stronger at the ITO edge in the active device region (corresponding to $y_{0}$ above 120), which is not very clearly discernible from the intensity image alone.

Figure 2(e) shows the color-coded images of the lifetime parameters $\left(\tau_{1}\right.$ and $\left.\tau_{2}\right)$ and their distribution among pixels at 0 $\mathrm{V}$. It is interesting to note that the distribution of $\tau_{1}$ has two distinct peaks around $0.7 \mathrm{~ns}$ (blue region) and 6-6.5 ns (green region), and these two peaks correspond to QDs in two regions in the scan area as seen from the color-coded image of $\tau_{1}$. The blue region, the fast PL lifetime $\mathrm{QD}$ region, corresponds to the quadrants III (active device) and IV, which all have an $\mathrm{Al}$ electrode on top of the other device layers. The quadrants I and II, which do not have an $\mathrm{Al}$ electrode, show $\tau_{1}$ with the peak around the 6-6.5 ns region. On the other hand, the $\tau_{2}$ distribution in the entire scan area has a single peak around 7 ns (yellow-green region), showing the uniform decay rate $\tau_{2}$ for the entire scan area. In fact for the QDs in quadrants I and II, the $\tau_{1}$ and $\tau_{2}$ distributions overlap each other effectively, implying a single decay lifetime that is similar to $\tau_{2}$ of the QDs in the quadrants III and IV. Thus, we can infer that the $\tau_{2}$ corresponds to the intrinsic decay lifetime of the QDs inside the device, and the fast lifetime $\tau_{1}$ is induced in the QDs inside the device due to the effect of the $\mathrm{Al}$ layer. It could be the result of the exciton quenching effect caused by the Al metallic electrode or some damage induced on the QDs while depositing Al. As will be shown later, this fast lifetime comes from QDs having a lower quantum efficiency. Considering that the thickness of intermediate layers between the $\mathrm{QDs}$ and $\mathrm{Al}$ on top is about $60-70 \mathrm{~nm}$, it is unlikely that this lifetime is induced by the quenching of excitons in QDs due to plasmonic effects. It is more probable that a layer of QDs is partially damaged during deposition of $\mathrm{Al}$ on top as the electrode. This is possible because the deposition of $\mathrm{Al}$ using thermal evaporation techniques requires heating to high temperatures to evaporate it. This heating effect probably leads to reduction 
in their quantum yield and lower efficiency of device performance. We performed a separate experiment to test the effect of depositing aluminum by measuring the decay lifetime distributions of QDs in two structures: in one we spin coated a QD layer on glass and thermally evaporated the CBP and aluminum layers ( $\mathrm{QD} / \mathrm{CBP} / \mathrm{Al}$ ) on top of it to make it similar to the device layers; in the other we first deposited the thermally evaporated layers of aluminum and $\mathrm{CBP}$ on glass and then spin coated the $\mathrm{QD}$ layer on it $(\mathrm{Al} / \mathrm{CBP} / \mathrm{QD})$ so that the effect of thermal evaporation on the $\mathrm{QD}$ layer is not present. Our results are shown in more detail in Supplementary Figure S2. We found that the fast lifetime value of $\tau_{1}$ (centered around $\sim 0.7 \mathrm{~ns}$ ) occurs again only in the $\mathrm{QD} / \mathrm{CBP} / \mathrm{Al}$ structure, whereas it is not present in the other. This reassures us that the low value of the $\tau_{1}$ lifetime component is occurring in our QLED due to the effect of thermal evaporation of Al. A lower temperature technique to deposit the metallic electrode such as the sputtering method might reduce the heating effect. Also, a reduced $\mathrm{Al}$ deposition rate using thermal evaporation or other techniques might be useful.

In the subsequent discussion, we analyze the effect of applying bias on the exciton PL decay lifetime starting from $0 \mathrm{~V}$ going up to $3.5 \mathrm{~V}$ when the device is sufficiently electroluminescent but still not very bright to cause any nonlinear effects on the PL single-photon counting. The device starts slightly emitting at $3.1 \mathrm{~V}$ and becomes fully electroluminescent quickly with increasing bias. The electroluminescence is captured in the TCSPC system as a background emission, which is different from the pulsed excitation and emission for time-resolved PL analysis. It is reflected as the fit parameter $y_{0}$ in the decay curves. We choose an area well within the active device region, away from the device edges, for all further analyses as shown in Figure 3(a) to obtain a qualitative understanding of the exciton decay especially under QLED operating conditions. Figure 3(a) shows the color-coded images of the $y_{0}$ parameter at different biases in the selected area of the active device region and its distribution across the pixels. As one can clearly see, the background $\left(y_{0}\right)$ is constant and has almost uniform distribution until $3 \mathrm{~V}$, after which it starts increasing together with the increase of electrical current. This increasing background light intensity is associated with device electroluminescence.

Figure 3(b) presents the distribution and color-coded images of the lifetime $\tau_{1}$ at different biases, which is the fast lifetime component induced by $\mathrm{Al}$ deposition as can be recalled from the previous discussion. We can see that $\tau_{1}$ is almost uniform in the device area, and its central distribution peak is around 0.7 $\mathrm{ns}$ with an fwhm of around $0.25 \mathrm{~ns}$ at different biases. Such fast decay lifetimes usually occur due to activation of fast nonradiative channels, which might indicate some damage caused to the $\mathrm{QDs}$ during $\mathrm{Al}$ deposition. Also, the distribution does not show much change on applying the bias, another evidence of the physical damage of the QDs instead of active quenching of excitons by other mechanisms. In contrast to $\tau_{1}$ in Figure $3(\mathrm{c})$, the $\tau_{2}$ decay lifetime, having the central distribution peak at around $7.3 \mathrm{~ns}$ (fwhm around $0.8 \mathrm{~ns}$ ) at 0 $\mathrm{V}$, is almost uniformly distributed in the entire device area. With the increase in bias starting from $3.0 \mathrm{~V}$, there is a systematic shift in the central value of $\tau_{2}$ distribution toward shorter lifetime accompanied by an increase in the spread of lifetime distribution. The shift of $\tau_{2}$ has "turn-on" behavior as the electrical current is injected into the device. The turn-on voltage for both $\tau_{2}$ shift, and the electrical current happens at around $3.0 \mathrm{~V}$. At $3.5 \mathrm{~V}$, the $\tau_{2}$ lifetime is faster with a central distribution peak around $6.6 \mathrm{~ns}$ and an fwhm of around $1.5 \mathrm{~ns}$. This indicates that electrical current through the device rather than the effect of QCSE causes an enhancement of the fast decay channels, which leads to a reduction in the $\tau_{2}$ lifetimes. The nature of these decay channels becomes further clear from Figure 3(d), which shows the total photoluminescence photon count taken from the selected area of the active device region at different biases. The detailed intensity images of the entire scan area for the fit parameters $y_{0}, \tau_{1}$, and $\tau_{2}$ at different biases are shown in Figure S3 in the Supporting Information. The photon count corresponds to the area below the decay curves after removing the constant intensity $y_{0}$. This can be computed by taking the sum of the product of amplitude and lifetime fit parameters $\left(A_{1} \tau_{1}+A_{2} \tau_{2}\right)$. This PL photon count is actually a reflection of the quantum efficiency of the QD ensemble with two different recombination channels.

Figure $3(d)$ shows the change in the contribution of each lifetime component to the total photon count with the application of bias. As can be seen, the total PL photon count decreases with increasing bias, which is predominantly due to the decrease in the contribution from the lifetime $\tau_{2}$ component. The contribution from the $\tau_{2}$ component at $0 \mathrm{~V}$ is about $90 \%$ of the total intensity contribution. This confirms that the lifetime $\tau_{1}$ is predominantly occurring from QDs with fast nonradiative channels. Since the photon count from the $\tau_{1}$ component also remains nearly unchanged with bias, this observation reinforces our earlier discussion that these are coming from $\mathrm{QDs}$ that might be physically damaged during $\mathrm{Al}$ deposition. The reduction in $\tau_{2}$ with increasing bias is also due to increasing of fast nonradiative channels in the QDs. With an increase of bias voltage, electrons and holes start to get injected into the QD layer, and their recombination in QDs generates spontaneous emission photons. These two types of charge are injected differently through their corresponding charge injection layers, and the imbalance of these injections would ionize the QDs. The decrease of $\tau_{2}$ with bias implies that the charge imbalance in the QDs induces fast nonradiative recombination of excitons with Auger-like mechanisms. The broadening of the lifetime distributions also suggests the spreading of the nonradiative channels among the QD ensemble.

Figure 3(e) shows the intensity-weighted average lifetime $\left(\tau_{\mathrm{i}}\right)$ of the two lifetimes in the specified area of active device region at different times during the experiment. It is given by $\tau_{\mathrm{i}}$ $=\left(A_{1} \tau_{1}^{2}+A_{2} \tau_{2}^{2}\right) /\left(A_{1} \tau_{1}+A_{2} \tau_{2}\right)$ and is considered to be a measure of the apparent lifetime ${ }^{35}$ of the $\mathrm{QD}$ excitons when using a multiexponential decay fit. At the start of the experiment, the $\tau_{\mathrm{i}}$ lifetime distribution at $0 \mathrm{~V}$ is centered at $6.6 \mathrm{~ns}$, which decreases to around $5.6 \mathrm{~ns}$ at $3.5 \mathrm{~V}$. By measuring the lifetimes upon applying $0 \mathrm{~V}$ again immediately after $3.5 \mathrm{~V}$, we find the lifetime shifts back slightly toward its position at the start of the experiment. This shows that, upon removing the bias, there is a slight recovery of the exciton lifetimes toward its original values; however there is a residual effect of the bias, possibly due to retained charges in the QDs, which prevent the full recovery of the lifetime to its original value at the start of the experiment. This also agrees with our previous discussion that the reduction in the exciton lifetime during device operation is due to the charging of QDs. Further, another measurement that was carried out without applying any bias on the device after a long time $(>3 \mathrm{~h})$ shows that the exciton lifetime still further recovers, but not fully. The result indicates 
that the charging of the device after operation might take a very long time to subside or there might be some physical QD damage during the operation.

To validate our measurement approach in an active device, it may be worth discussing our TCSPC technique to obtain the exciton lifetime in our experiments. The reduced chi-square $\left(\chi_{\mathrm{r}}^{2}\right)$ distribution for all the fitting is close to 1 , as shown in Supplementary Figure S4. Our photon detection probability (characteristic of our TCSPC setup) is small enough to avoid any distortion effects in our range of experiments. However, the lifetime measurement under high constant illumination might distort the PL decay curves and result in different implications of exciton lifetime fitting. To investigate this effect, we did a separate series of experiments using FLIM measurement for a plain QD film on glass while monitoring and controlling the background illumination intensity with a lamp during measurement. Here the counting rate was controlled to be similar to the experiments with QLEDs under different biases. Our results are shown in the Supporting Information, Figure S5. There is indeed an artificial distortion of PL decay curves, producing an increase in measured lifetime distribution peaks and fwhm's with increasing background intensity. This is absolutely opposite of our results with the QLEDs, in which QD decay lifetimes decrease with increasing background constant intensity $\left(y_{0}\right)$ caused by the electroluminescence of the device. This result reassures that the observed decrease of exciton lifetimes with increasing voltage bias is indeed the intrinsic properties of QDs in QLEDs, and the real effect is even larger. The artificial effect of increasing background illumination intensity on the lifetime distribution fwhm, however, cannot be completely ruled out.

\section{CONCLUSION}

In this work, we presented the effect of bias voltage on the exciton dynamics in a working QLED device fabricated using the gradient composition CdSe@ZnS QDs. By analyzing the time-resolved PL decays of many QDs distributed on QLED devices, we understand their changing exciton behavior with a statistically large population. Besides QY reduction of the emissive QD layer due to device structure and fabrication, charge imbalance caused by the current injection during the device operation reduces the $\mathrm{QY}$ and lowers the efficiency of a QLED. Our detailed investigation reveals that the QD efficiency decrease in QLED devices at increased current densities is due to QD-charging-induced Auger recombination, even in gradient composition CdSe@ZnS QDs.

\section{METHODS}

Synthesis of CdSe@ZnS QDs. CdSe@ZnS QDs were synthesized according to a modified method reported in the literature. ${ }^{34}$ For a typical preparation, $0.4 \mathrm{mmol}$ of cadmium oxide $(\mathrm{CdO}), 4 \mathrm{mmol}$ of zinc acetate $\left(\mathrm{Zn}(\text { Acet })_{2}\right)$, and $5 \mathrm{~mL}$ of oleic acid (OA) were mixed and heated to $100{ }^{\circ} \mathrm{C}$ under vacuum in a $50 \mathrm{~mL}$ three-neck flask. Then, $15 \mathrm{~mL}$ of 1 octadecene (1-ODE) was injected, and the whole mixture was degassed again to $100{ }^{\circ} \mathrm{C}$. Then the reactor was filled with argon and further heated to $310^{\circ} \mathrm{C}$. At this temperature, $2 \mathrm{~mL}$ of tri-n-octylphosphine (TOP) with $0.2 \mathrm{mmol}$ of selenium (Se) and $4 \mathrm{mmol}$ of sulfur (S) was injected into the flask swiftly. The reaction was kept at $310^{\circ} \mathrm{C}$ for $10 \mathrm{~min}$ for the $\mathrm{QD}$ growth. For purification, excess acetone and methanol were added to precipitate the QDs, followed by centrifugation at a speed of
$7000 \mathrm{rpm}$ for $10 \mathrm{~min}$. The purified QDs were dispersed in toluene for later use.

Synthesis of ZnO Nanoparticles. For a typical synthesis of $\mathrm{ZnO}$ nanoparticles, $3 \mathrm{mmol}$ of zinc acetate (anhydrous) was dissolved in $30 \mathrm{~mL}$ of dimethyl sulfoxide (DMSO). An ethanol solution of $10 \mathrm{~mL}$ dissolved with $5 \mathrm{mmol}$ of tetramethylammonium hydroxide (TMAH) was dropwise introduced to the above $\mathrm{Zn}$ solution and stirred for $1 \mathrm{~h}$ under ambient conditions. The $\mathrm{ZnO}$ nanoparticles were precipitated with an excess amount of acetone and then completely redispersed in ethanol. The solutions were filtered before use.

Measurements and Fabrication. All device fabrication was carried out using standard procedures, a combination of spin-casting and thermal vapor deposition in a vacuum chamber. The voltage-current measurements were performed using a Yokagawa GS610 source meter. The luminance measurements were taken using a Minolta LS110 luminance meter.

The time-resolved PL spectroscopy was performed with a Becker \& Hickl DCS 120 confocal scanning FLIM system with an excitation laser of $375 \mathrm{~nm}$. The system has a temporal resolution of 200 ps. For all the time-resolved PL measurements, photons were collected in $300 \mathrm{~s}$.

\section{ASSOCIATED CONTENT}

\section{S Supporting Information}

The Supporting Information is available free of charge on the ACS Publications website at DOI: 10.1021/acsphotonics.7b00984.

TEM image of CdSe@ZnS QDs; FLIM measurement results of $\mathrm{QDs}$ in $\mathrm{QD} / \mathrm{CBP} / \mathrm{Al}$ and $\mathrm{Al} / \mathrm{CBP} / \mathrm{QD}$ structures; intensity and color-coded images of fitting parameters for the entire scan area at different biases; $\chi_{\mathrm{r}}^{2}$ distribution for fitting at different biases; FLIM measurement results of plain QD film on glass under different background illumination intensities (PDF)

\section{AUTHOR INFORMATION}

\section{Corresponding Authors}

*E-mail: hcdang@ntu.edu.sg.

*E-mail: volkan@bilkent.edu.tr, hvdemir@ntu.edu.sg.

ORCID

Sushant Shendre: 0000-0001-8586-7145

Vijay Kumar Sharma: 0000-0002-2028-5715

Cuong Dang: 0000-0001-6183-4082

Hilmi Volkan Demir: 0000-0003-1793-112X

\section{Author Contributions}

The manuscript was written through contributions of all authors. All authors have given approval to the final version of the manuscript.

\section{Notes}

The authors declare no competing financial interest.

\section{ACKNOWLEDGMENTS}

This research is supported by the National Research Foundation, Prime Minister's Office, Singapore, under its NRF Investigatorship Award Program (NRF-NRFI2016-08) and the Singapore Agency for Science, Technology and Research (A*STAR) SERC Pharos Program under Grant No. 15273 00025. H.V.D. gratefully acknowledges TUBA-GEBIP, 
and C.D. acknowledges support from the Nanyang Technological University start-up grant M4081482.

\section{REFERENCES}

(1) Supran, G. J.; Shirasaki, Y.; Song, K. W.; Caruge, J.-M.; Kazlas, P. T.; Coe-Sullivan, S.; Andrew, T. L.; Bawendi, M. G.; Bulović, V. QLEDs for Displays and Solid-State Lighting. MRS Bull. 2013, 38, 703-711.

(2) Shirasaki, Y.; Supran, G. J.; Bawendi, M. G.; Bulovic, V. Emergence of Colloidal Quantum-Dot Light-Emitting Technologies. Nat. Photonics 2013, 7, 13-23.

(3) Erdem, T.; Demir, H. V. Color Science of Nanocrystal Quantum Dots for Lighting and Displays. Nanophotonics 2013, 2, 57-81.

(4) Demir, H. V.; Nizamoglu, S.; Erdem, T.; Mutlugun, E.; Gaponik, N.; Eychmüller, A. Quantum Dot Integrated LEDs Using Photonic and Excitonic Color Conversion. Nano Today 2011, 6, 632-647.

(5) Pietryga, J. M.; Park, Y.-S.; Lim, J.; Fidler, A. F.; Bae, W. K.; Brovelli, S.; Klimov, V. I. Spectroscopic and Device Aspects of Nanocrystal Quantum Dots. Chem. Rev. 2016, 116, 10513-10622.

(6) Dai, X.; Deng, Y.; Peng, X.; Jin, Y. Quantum-Dot Light-Emitting Diodes for Large-Area Displays: Towards the Dawn of Commercialization. Adv. Mater. 2017, 29, 1607022.

(7) Yang, X.; Mutlugun, E.; Dang, C.; Dev, K.; Gao, Y.; Tan, S. T.; Sun, X. W.; Demir, H. V. Highly Flexible, Electrically Driven, TopEmitting, Quantum Dot Light-Emitting Stickers. ACS Nano 2014, 8, 8224-8231.

(8) Kwak, J.; Bae, W. K.; Lee, D.; Park, I.; Lim, J.; Park, M.; Cho, H.; Woo, H.; Yoon, D. Y.; Char, K. Bright and Efficient Full-Color Colloidal Quantum Dot Light-Emitting Diodes Using an Inverted Device Structure. Nano Lett. 2012, 12, 2362-2366.

(9) Mashford, B. S.; Stevenson, M.; Popovic, Z.; Hamilton, C.; Zhou, Z.; Breen, C.; Steckel, J.; Bulovic, V.; Bawendi, M.; Coe-Sullivan, S. High-Efficiency Quantum-Dot Light-Emitting Devices with Enhanced Charge Injection. Nat. Photonics 2013, 7, 407-412.

(10) Dai, X.; Zhang, Z.; Jin, Y.; Niu, Y.; Cao, H.; Liang, X.; Chen, L.; Wang, J.; Peng, X. Solution-Processed, High-Performance LightEmitting Diodes Based on Quantum Dots. Nature 2014, 515, 96-9.

(11) Dang, C.; Lee, J.; Breen, C.; Steckel, J. S.; Coe-Sullivan, S.; Nurmikko, A. Red, Green and Blue Lasing Enabled by Single-Exciton Gain in Colloidal Quantum Dot Films. Nat. Nanotechnol. 2012, 7, 335-339.

(12) Dang, C.; Lee, J.; Roh, K.; Kim, H.; Ahn, S.; Jeon, H.; Breen, C.; Steckel, J. S.; Coe-Sullivan, S.; Nurmikko, A. Highly Efficient, Spatially Coherent Distributed Feedback Lasers from Dense Colloidal Quantum Dot Films. Appl. Phys. Lett. 2013, 103, 171104.

(13) Dang, C.; Nurmikko, A. Beyond Quantum Dot LEDs: Optical Gain and Laser Action in Red, Green, and Blue Colors. MRS Bull. 2013, 38, 737-742.

(14) Qin, H.; Niu, Y.; Meng, R.; Lin, X.; Lai, R.; Fang, W.; Peng, X. Single-Dot Spectroscopy of Zinc-Blende CdSe/CdS Core/Shell Nanocrystals: Nonblinking and Correlation with Ensemble Measurements. J. Am. Chem. Soc. 2014, 136, 179-187.

(15) Galland, C.; Ghosh, Y.; Steinbrück, A.; Hollingsworth, J. A.; Htoon, H.; Klimov, V. I. Lifetime Blinking in Nonblinking Nanocrystal Quantum Dots. Nat. Commun. 2012, 3, 908.

(16) Park, Y.-S.; Bae, W. K.; Pietryga, J. M.; Klimov, V. I. Auger Recombination of Biexcitons and Negative and Positive Trions in Individual Quantum Dots. ACS Nano 2014, 8, 7288-7296.

(17) Guzelturk, B.; Martinez, P. L. H.; Zhang, Q.; Xiong, Q.; Sun, H.; Sun, X. W.; Govorov, A. O.; Demir, H. V. Excitonics of Semiconductor Quantum Dots and Wires for Lighting and Displays. Laser Photonics Rev. 2014, 8, 73-93.

(18) Gao, Y.; Peng, X. Photogenerated Excitons in Plain Core CdSe Nanocrystals with Unity Radiative Decay in Single Channel: The Effects of Surface and Ligands. J. Am. Chem. Soc. 2015, 137, 42304235.

(19) Bae, W. K.; Lim, J.; Zorn, M.; Kwak, J.; Park, Y.-S.; Lee, D.; Lee, S.; Char, K.; Zentel, R.; Lee, C. Reduced Efficiency Roll-Off in Light-
Emitting Diodes Enabled by Quantum Dot-Conducting Polymer Nanohybrids. J. Mater. Chem. C 2014, 2, 4974-4979.

(20) Minotto, A.; Todescato, F.; Fortunati, I.; Signorini, R.; Jasieniak, J. J.; Bozio, R. Role of Core-Shell Interfaces on Exciton Recombination in CdSe- $\mathrm{Cd}_{x} \mathrm{Zn}_{1-x} \mathrm{~S}$ Quantum Dots. J. Phys. Chem. C 2014, 118, 24117-24126.

(21) Park, Y.-S.; Bae, W. K.; Padilha, L. A.; Pietryga, J. M.; Klimov, V. I. Effect of the Core/Shell Interface on Auger Recombination Evaluated by Single-Quantum-Dot Spectroscopy. Nano Lett. 2014, 14, 396-402.

(22) Garcia-Santamaria, F.; Chen, Y. F.; Vela, J.; Schaller, R. D.; Hollingsworth, J. A.; Klimov, V. I. Suppressed Auger Recombination in "Giant" Nanocrystals Boosts Optical Gain Performance. Nano Lett. 2009, 9, 3482-3488.

(23) Lim, J.; Jeong, B. G.; Park, M.; Kim, J. K.; Pietryga, J. M.; Park, Y.-S.; Klimov, V. I.; Lee, C.; Lee, D. C.; Bae, W. K. Influence of Shell Thickness on the Performance of Light-Emitting Devices Based on $\mathrm{CdSe} / \mathrm{Zn}_{1-\mathrm{X}} \mathrm{Cd}_{\mathrm{X}} \mathrm{S}$ Core/Shell Heterostructured Quantum Dots. Adv. Mater. 2014, 26, 8034-8040.

(24) Anikeeva, P.; Madigan, C.; Halpert, J.; Bawendi, M.; Bulović, V. Electronic and Excitonic Processes in Light-Emitting Devices Based on Organic Materials and Colloidal Quantum Dots. Phys. Rev. B: Condens. Matter Mater. Phys. 2008, 78, 085434.

(25) Bae, W. K.; Brovelli, S.; Klimov, V. I. Spectroscopic Insights into the Performance of Quantum Dot Light-Emitting Diodes. MRS Bull. 2013, 38, 721-730.

(26) Bozyigit, D.; Wood, V. Challenges and Solutions for HighEfficiency Quantum Dot-Based LEDs. MRS Bull. 2013, 38, 731-736.

(27) Shirasaki, Y.; Supran, G. J.; Tisdale, W. A.; Bulović, V. Origin of Efficiency Roll-Off in Colloidal Quantum-Dot Light-Emitting Diodes. Phys. Rev. Lett. 2013, 110, 217403.

(28) Bozyigit, D.; Yarema, O.; Wood, V. Origins of Low Quantum Efficiencies in Quantum Dot LEDs. Adv. Funct. Mater. 2013, 23, 3024-3029.

(29) Bae, W. K.; Park, Y.-S.; Lim, J.; Lee, D.; Padilha, L. A.; McDaniel, H.; Robel, I.; Lee, C.; Pietryga, J. M.; Klimov, V. I. Controlling the Influence of Auger Recombination on the Performance of Quantum-Dot Light-Emitting Diodes. Nat. Commun. 2013, 4, 2661.

(30) Vaxenburg, R.; Lifshitz, E.; Efros, A. L. Suppression of Augerstimulated efficiency droop in nitride-based light emitting diodes. Appl. Phys. Lett. 2013, 102, 031120.

(31) Iveland, J.; Martinelli, L.; Peretti, J.; Speck, J. S.; Weisbuch, C. Direct Measurement of Auger Electrons Emitted from a Semiconductor Light-Emitting Diode under Electrical Injection: Identification of the Dominant Mechanism for Efficiency Droop. Phys. Rev. Lett. 2013, 110, 177406.

(32) Cragg, G. E.; Efros, A. L. Suppression of Auger Processes in Confined Structures. Nano Lett. 2010, 10, 313-317.

(33) Yang, Y.; Zheng, Y.; Cao, W.; Titov, A.; Hyvonen, J.; Manders, J. R; Xue, J.; Holloway, P. H.; Qian, L. High-Efficiency Light-Emitting Devices Based on Quantum Dots with Tailored Nanostructures. Nat. Photonics 2015, 9, 259-266.

(34) Bae, W. K.; Char, K.; Hur, H.; Lee, S. Single-Step Synthesis of Quantum Dots with Chemical Composition Gradients. Chem. Mater. 2008, 20, 531-539.

(35) Lakowicz, J. R. Principles of Fluorescence Spectroscopy; Springer Science and Business Media, LLC: New York, 2006; pp 97-149. 